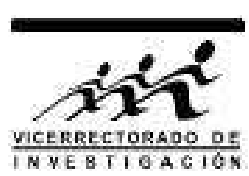

\title{
Formulación ADM de la Relatividad General
}

\author{
Fulgencio Villegas Silva ${ }^{*}$ T Teófilo Vargas Auccalla* ${ }^{* *}$ \\ Facultad de Ciencias Físicas, Universidad Nacional Mayor de San Marcos, Lima, Perú
}

Recibido 20 noviembre 2016 - Aceptado 15 diciembre 2016

\begin{abstract}
Se realiza el estudio del formalismo ADM para la Teoría de la Relatividad General mediante el método de las foliaciones en las superficies de Cauchy. Se presenta explícitamente el desarrollo matemático que conduce, finalmente, a la formulación hamiltoniana de la Relatividad General.

Palabras claves: Relatividad General, gravitación, relatividad numérica, formalismo ADM.
\end{abstract}

\section{ADM formulation of the General Relativity}

The study of the ADM formalism for the General Relativity Theory through the foliation methods on the Cauchy surfacesis is realized. The mathematical development that leads to the Hamiltonian formulation of General Relativity is presented explicitly.

Keywords: General Relativity, gravitation, numerical relativity, ADM formalism.

Las ecuaciones de la Relatividad General de Einstein determinan la evolución de los sistemas gravitacionales, siendo uno de los objetivos fundamentales la determinación de la evolución temporal del campo gravitacional de los sistemas estudiados. Sin embargo, los sistemas que poseen soluciones exactas y analíticas son pocos y la mayoría de ellos son simples ya que presentan ciertas simetrías que contribuyen a obtener dichas soluciones. Para sistemas reales o mas complejos se hace necesario optar por otros métodos de solución. En la actualidad existen dos métodos alternativos, uno de ellos es la solución numérica y el otro método esta basado en los procedimientos perturbativos. Para obtener soluciones numéricas a las ecuaciones de la Relatividad General de Einstein se deben transformar las ecuaciones de campo de Einstein a un problema de Cauchy o problema de valor inicial también conocido como el formalismo $3+1$ [1].

La formulación hamiltoniana del campo gravitacional de Einstein aparece como un instrumento privilegiado para estudiar la evolución del espacio-tiempo ya que describe la evolución de la geometría tri-dimensional en el tiempo; esta formulación fue estudiada en sus inicios por Dirac [2], posteriormente por Arnowitt, Deser y Misner [3] cuya metodología es conocida como el formalismo ADM, acrónimo formado por las iniciales de sus nombres. La importancia de la formulación hamiltoniana de la Relatividad General radica en que es el primer paso para la cuantización canónica del espacio-tiempo, que Wheleer lo denominó como la geometrodinámica cuántica [4].

\footnotetext{
${ }^{*}$ fvillegass@unmsm.edu.pe

** teofilo.vargas@gmail.com
}

En este trabajo, hacemos una revisión de los conceptos fundamentales de la mecánica clásica, Relatividad General y de la descomposición $3+1$ mediante el método de las foliaciones de una variedad diferenciable, para finalmente obtener las variables ADM que conducen a la expresión del hamiltoniano de la Relatividad General en función de dichas variables.

\section{La acción clásica}

Las ecuaciones que gobiernan la evolución de los campos clásicos son las ecuaciones de Euler-Lagrange las cuales son obtenidas por medio de un principio variacional.

Considerando que la densidad lagrangiana $\mathcal{L}$ depende del campo $\phi$ y de sus primeras derivadas $\mathcal{L}=\mathcal{L}\left(\phi, \partial_{\mu} \phi\right)$. La acción solemos usualmente escribirla como [5]

$$
S=\int \mathcal{L}\left(\phi, \partial_{\mu} \phi\right) d^{4} x
$$

Por el principio de mínima acción $S$ debe ser estacionaria para toda variación de $\phi, \delta \phi$, que se anule en la frontera de una región; por tanto debe satisfacer $\delta S=0$, o equivalentemente

$$
\frac{\partial \mathcal{L}}{\partial \phi}-\partial_{\mu}\left(\frac{\partial \mathcal{L}}{\partial\left(\partial_{\mu} \phi\right)}\right)=0
$$

cuya expresión es conocida como las ecuaciones de campo o de Euler-Lagrange. 
Definimos el momentum conjugado como

$$
\pi=\frac{\partial \mathcal{L}}{\partial \dot{\phi}}
$$

donde $\cdot=\frac{\partial}{\partial t}$.

Por lo tanto, la densidad hamiltoniana se define como

$$
\mathcal{H}(\pi, \phi)=\pi \dot{\phi}-\mathcal{L}
$$

donde el hamiltoniano está dado por la integral en el espacio de su densidad

$$
H=\int d^{4} x \mathcal{H}
$$

Haciendo uso de la ecuación (4) se obtienen las ecuaciones de Hamilton

$$
\dot{\pi}=-\frac{\partial \mathcal{H}}{\partial \phi}, \quad \dot{\phi}=\frac{\partial \mathcal{H}}{\partial \pi}, \quad \frac{d \mathcal{H}}{d t}=-\frac{\partial \mathcal{L}}{\partial t} .
$$

Las cantidades $\pi$ y $\phi$ juntas, definen el espacio de fase que describe el campo clásico y su desarrollo en el tiempo.

\section{Principios y postulados de la Relatividad Ge- neral}

La Teoría de la Relatividad General es la teoría moderna de la gravitación, fue postulada por Einstein a finales de 1915. Según esta teoría, un objeto masivo produce una distorsión o curvatura en el espacio-tiempo, siendo la gravitación una manifestación de esta distorsión [6].

Los principios que guiaron a Einstein a la formulación de la Relatividad General fueron:

- El principio de covariancia establece que las leyes de la física deben ser las mismas para todos los observadores, o todos los observadores son equivalentes. Este principio llevó a Einstein a considerar que las leyes físicas deberían escribirse en forma tensorial.

- El principio de equivalencia establece que todos los objetos caen de la misma forma en un campo gravitacional, es decir, que las leyes de la relatividad especial se aplican localmente para todos los observadores inerciales. En base a este principio, Einstein concluyó que la descripción de la gravedad debería ser identificada con la geometría del espacio-tiempo.

- El principio de Mach establece que la inercia local de un objeto debe ser producida por la distribución total de la materia en el universo. Este principio llevó a Einstein a concluir que la geometría del espacio-tiempo debe ser alterada por la distribución de materia.

La Teoría General de la Relatividad es una teoría geométrica de la la gravitación, su construcción se basa en una serie de postulados, los cuales fueron desarrollados buscando compatibilidad con el límite newtoniano y con la Relatividad Especial.
Postulado 1. El espacio tiempo está descrito por el par $(M, g)$ siendo $M$ una variedad 4-dimensional y $g$ una métrica Lorentziana sobre $M$.

Este postulado se basa en la descripción del espaciotiempo, donde la curvatura en la variedad diferenciable está determinada por el tensor de la curvatura de Riemann que escrito como componentes tiene la forma

$$
R_{\beta \gamma \delta}^{\alpha}=\partial_{\gamma} \Gamma_{\delta \beta}^{\alpha}-\partial_{\delta} \Gamma_{\gamma \beta}^{\alpha}+\Gamma_{\gamma \sigma}^{\alpha} \Gamma_{\delta \beta}^{\sigma}-\Gamma_{\sigma \delta}^{\alpha} \Gamma_{\gamma \beta}^{\sigma},
$$

donde $\Gamma_{\beta \gamma}^{\alpha}$ son los símbolos de Christoffel, los cuales vienen expresados por

$$
\Gamma_{\beta \gamma}^{\alpha}=\frac{1}{2} g^{\alpha \sigma}\left[\partial_{\sigma} g_{\alpha \sigma}+\partial_{\beta} g_{\sigma \gamma}-\partial_{\sigma} g_{\beta \gamma}\right],
$$

en donde $g_{\alpha \beta}$ son las componentes del tensor métrico.

El tensor de Riemann sirve para caracterizar la geometría de la variedad $M$. En particular, representa una indicación sobre la curvatura de la misma, o en otras palabras, expresa el hecho de que tan paralelamente es posible transportar un vector a lo largo de una curva. Por esta razón el único indicador de la curvatura del espacio-tiempo es el tensor de la curvatura. Si todas las componentes del tensor de la curvatura son nulas, entonces, el espacio-tiempo es plano y si alguna de sus componentes es diferente de cero, entonces, el espacio-tiempo es curvo.

Postulado 2. Existe un tensor simétrico $T_{\alpha \beta}=T_{\alpha \beta}(\varphi)=$ $T_{\beta \alpha}$ que es función de los campos de materia $\varphi$ y sus derivadas tal que:

1. $T_{\alpha \beta}=0$ sobre $\mathcal{U} \subset M$ si y solo si $\varphi_{i}=0$ para todo $i$ sobre $\mathcal{U}$.

2. $\nabla_{\beta} T^{\alpha \beta}=0$.

Este postulado esta referido a la conservación de la energía la cual juega un papel fundamental en la Relatividad General.

Postulado 3. La métrica sobre la variedad espacio-tiempo $(M, g)$ está determinada por las ecuaciones de campo de Einstein.

Las ecuaciones de campo de Einstein está dado por

$$
R_{\alpha \beta}-\frac{1}{2} R g_{\alpha \beta}=k T_{\alpha \beta},
$$

donde $R_{\alpha \beta}$ es el tensor de Ricci , $R$ es el escalar de curvatura, $T_{\alpha \beta}$ el tensor de energía momentum, y $k=8 \pi G, G$ la constante de gravitación universal.

El tensor de Einstein $G_{\alpha \beta}$ se define por la relación

$$
G_{\alpha \beta}=R_{\alpha \beta}-\frac{1}{2} R g_{\alpha \beta},
$$

la cual cumple con la siguiente propiedad

$$
\nabla_{\beta} G^{\alpha \beta}=0,
$$


siendo esta ecuación conocida como la Identidad de Bianchi.

Las ecuaciones de campo son obtenidas a partir de la acción de Einstein-Hilbert $\left(S_{E H}\right)$. Esta acción, que genera las ecuaciones de Einstein para el vacío, viene dada por

$$
S_{E H}\left(g_{\mu \nu}\right)=\frac{1}{2 k} \int d^{4} x \sqrt{-g} R, \quad \mu, \nu=0,1,2,3,
$$

donde $g$ es el determinante del tensor métrico y $R$ el escalar de curvatura. $S_{E H}$ es un funcional de $g_{\mu \nu}$.

Realizando una variación en la acción

$$
\begin{aligned}
& \delta S_{E H}=\int d^{4} x\left(\sqrt{-g} g^{\mu \nu} \delta R_{\mu \nu}\right.+\sqrt{-g} R_{\mu \nu} \delta g^{\mu \nu} \\
&\left.+g^{\mu \nu} R_{\mu \nu} \delta \sqrt{-g}\right) \\
& \delta S_{E H}=\int d^{4} x \sqrt{-g} \delta g^{\mu \nu}\left(R_{\mu \nu}-\frac{1}{2} R g_{\mu \nu}\right) .
\end{aligned}
$$

Considerando $\delta S_{E H}=0$, obtenemos

$$
R_{\mu \nu}-\frac{1}{2} R g_{\mu \nu}=0,
$$

que es la ecuación de Einstein en el vacío.

\section{Foliación 3+1 del espacio-tiempo y variables ADM}

Un camino directo para cuantizar la gravitación es la aplicación del formalismo canónico. Para ello es necesario reescribir la Relatividad General en una formulación dinámica, es decir, escribirla como un problema de valores iniciales o problema de Cauchy; sin embargo esto no es posible realizarlo directamente debido a que las ecuaciones de Einstein están escritas en forma covariante, del tal forma que el espacio y el tiempo juegan un papel de equivalencia. $\mathrm{Pa}-$ ra reescribir las ecuaciones de Eisntein como un problema de Cauchy se tiene que separar la variable temporal de las variables espaciales; a este proceso se le conoce como fromalismo ADM o formalismo 3+1 de la Relatividad General desarrollado por R.Arnowitt, S. Deser e C. W. Misner [3].

El espacio-tiempo es posible describirlo por medio de una variedad 4-dimensional $M$. Esta representación es posible descomponerla haciendo que una variedad 3dimensional $\Sigma$ sea dependiente de un parámetro real como el tiempo. Esto es posible debido al siguiente teorema:

Teorema 1. Sea $\left(M, g_{\mu \nu}\right)$ un espacio-tiempo globalmente hiperbólico, entonces una función global $t$ puede ser escogida tal que cada superficie de $t$ constante es una superficie de Cauchy. Por tanto $M$ puede ser foliada por superficies de Cauchy y la topología es $\mathbb{R} \times \Sigma$, donde $\Sigma$ denota cualquier superficie de Cauchy.

Es importante señalar que el espacio-tiempo cuadridimensional es globalmente hiperbólico si admite una subvariedad de tipo espacio que corta una y sola una vez a cada curva temporal, y esta sub-variedad se llama superficie de Cauchy. Esta condición de hiperbolicidad global garantiza el buen comportamiento causal de las diferentes soluciones de las ecuaciones de Einstein.

Del teorema anterior podemos identificar que las superficies $\Sigma_{t}$, para cada $t \in \mathbb{R}$ fijo, representan una familia de sub-espacios Riemannianos $\left(M^{3}, h_{\mu \nu}\right)$ de dimensión 3, dotados de una métrica inducida $h_{\mu \nu}$ sobre $M^{3}$. Dentro de este contexto podemos indicar que una variedad globalmente hiperbólica $M$ en el espacio-tiempo es descrita por su métrica $g_{\mu \nu}$ en este espacio; si dicho espacio-tiempo puede ser foliado de tal manera que cada folio, hoja o rebanada tridimensional sea de tipo espacio (space-like), entonces se dice que dicha variedad espacio-temporal es globalmente hiperbólica. Definimos el parámetro $t$ como aquel que identifica a las distintas hojas o rebanadas de la foliación; este parámetro $t$ es una etiqueta asignada a cada hipersuperficie $\Sigma_{t}$ y podemos dotarle de características temporales, considerándolo como un tiempo universal. La figura 1 nos da una noción mas intuitiva de como el proceso es empleado.

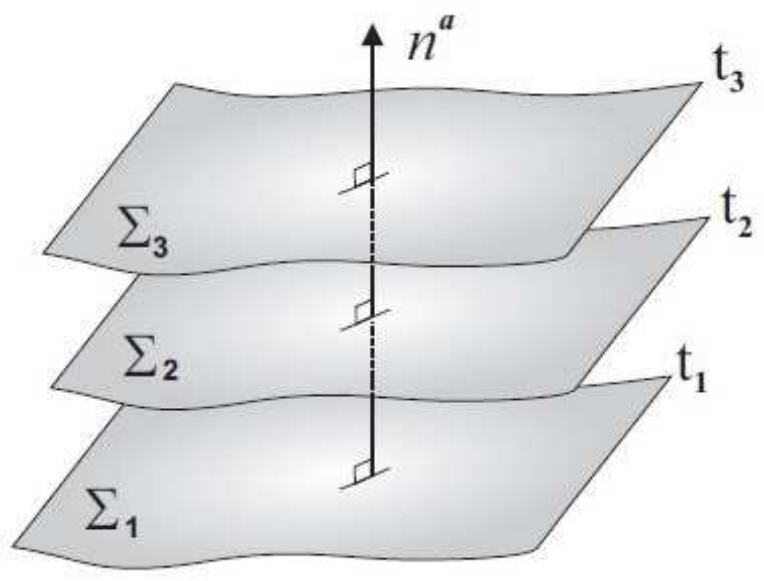

Figura 1: Foliación del espacio-tiempo en hipersuperficies espaciales tridimensionales.

Ahora consideremos un espacio-tiempo con una métrica $g_{\mu \nu}$, la cual posee 10 componentes independientes; de estas, solamente seis pueden ser calculadas a partir de las ecuaciones de Einstein. Las cuatro variables faltantes son definidas por la foliación del espacio-tiempo en hipersuperficies: una de ellas es la función lapso (lapse function) $N$ las otras tres son las funciones de desplazamiento (shift vector) $N^{\mu}$ el cual está definido sobre las hipersuperficies tridimensionales tipo espacio con métricas $h_{\mu \nu}$. La función lapso y el vector desplazamiento son cantidades que deben ser fijadas externamente y son ellas las que determinan la forma por la cual las hipersuperficies tri-dimensionales tipo espaciales evolucionan para dar origen al espacio-tiempo tetra-dimensional. 


\section{Función lapso}

A partir de la funcio $t$ podemos definir el vector normal al folio o rebanada $\Sigma_{t}$ como

$$
n^{\mu}=-N \nabla^{\mu} \operatorname{tcon} N>0,
$$

donde el signo " - " es debido a la signatura $(-,+,+,+)$ de $g_{\mu \nu}$. Se debe tener presente que $n^{\mu}$ es un vector normalizado tipo temporal, puesto que $n^{\mu} n_{\mu}=-1$ de tal manera que $n^{\mu}$ está orientado en el sentido de $t$ creciente. La función $N: M^{4} \longrightarrow \mathbb{R}$ denominada función lapso se introduce para normalizar a $n^{\mu}$, es decir,

$$
N=(-\nabla t . \nabla t)^{-1 / 2} .
$$

La función lapso nos informa la variación de tiempo propio cuando un observador se mueve entre dos folios vecinos siguiendo la trayectoria normal $n^{\mu}$ [7].

\section{Métrica inducida}

La métrica inducida $h_{\mu \nu}$ sobre $\Sigma_{t}$ mide las distancias $d s^{2}=h_{\mu \nu} d x^{\mu} d x^{\nu}$ dentro de la hipersuperficie misma, y esta definida por

$$
h_{\mu \nu}=g_{\mu \nu}+n_{\mu} n_{\nu}
$$

donde $g_{\mu \nu}$ es la métrica de $M^{4}$ y $n_{\mu}$ es el 4-vector normal a $\Sigma_{t}$.

La métrica inducida está contenida enteramente en la hipersuperficie tridimensional, por tanto $h_{\mu \nu} n^{\mu}=0$, siendo por tanto una cantidad puramente espacial. La métrica espacial inversa $h^{\mu \nu}$ es obtenida levantando los indices de $h_{\mu \nu} \operatorname{con} g^{\mu \nu}$,

$$
h^{\mu \nu}=g^{\alpha \mu} g^{\beta \nu} h_{\alpha \beta}=g^{\mu \nu}+n^{\mu} n^{\nu} .
$$

De igual manera, el operador de proyección que envía a un vector del espacio tiempo a uno sobre la hipersuperficie esta dado por

$$
h_{\nu}^{\mu}=\delta_{\nu}^{\mu}+n^{\mu} n_{\nu}
$$

\section{Vector desplazamiento}

Un observador puede moverse entre dos folios o rebanadas a lo largo de una dirección que no sea necesariamente la dirección normal $n^{\mu}$. El vector temporal que describe una trayectoria arbitraria esta dado por

$$
t^{\mu}=N n^{\mu}+N^{\mu}
$$

donde $N^{\mu}$ es el vector desplazamiento, que es el vector tangente al folio $\circ$ rebanada $\Sigma_{t}$ en que se encuentra situado. Definido $N^{\mu}$ de esta manera, entonces se tiene que $N^{\mu} n_{\mu}=0$.
Por lo tanto, el 4-vector desplazamiento $N^{\mu}$ se puede definir como la proyección de $t^{\nu}$ sobre $\Sigma_{t}$, de la forma

$$
N^{\mu}=-h_{\mu}^{\nu} t^{\nu}
$$

La relación entre el flujo temporal y los parámetros $N$ y $N^{\mu}$ se representan gráficamente en la figura 2 .

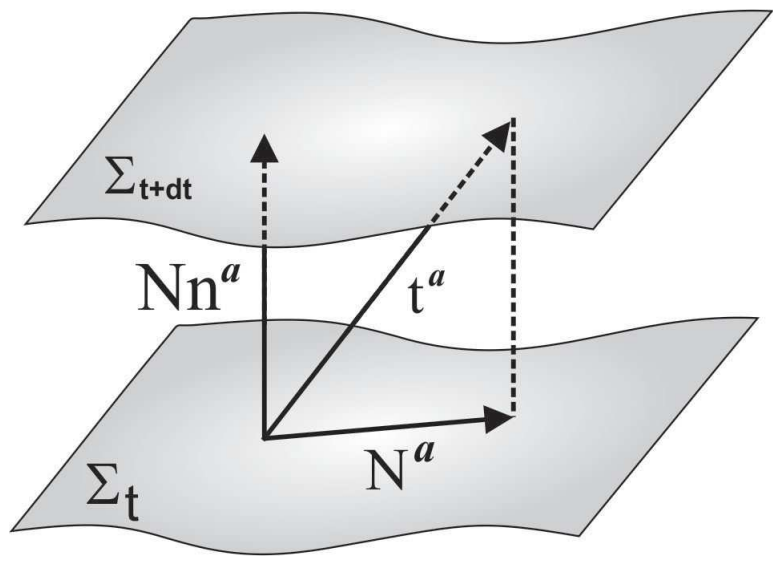

Figura 2: El vector de campo temporal puede ser escrito en función de la variable lapso y del vector de desplazamiento.

El escalar $N$, el vector sobre la hipersuperficie $N^{\mu}$ y la métrica inducida $h_{\mu \nu}$ conforman las llamadas variables ADM.

\section{Componentes de la métrica inducida}

Partiendo de las relaciones $n_{\mu}=(-N, 0,0,0), n^{t} n_{t}=$ -1 y $N^{\mu}=-h_{\nu}^{\mu} t^{\nu}$ se demuestra que las componentes del vector normal unitario $n^{\mu}$ a las hipersuperficies son

$$
\begin{aligned}
n_{\mu} & =(-N, 0,0,0), \\
n^{\mu} & =\left(\frac{1}{N}, \frac{N^{i}}{N}\right) \\
N^{\mu} & =\left(0, N^{i}\right) \\
t^{\mu} & =(1,0,0,0) .
\end{aligned}
$$

Haciendo uso de la ecuación (18) y de la ecuación (21) obtenemos

$$
g^{\mu \nu}=h^{\mu \nu}-\frac{1}{N^{2}}\left(t^{\mu}-N^{\mu}\right)\left(t^{\nu}-N^{\nu}\right) .
$$

Desde luego que $x^{0}=t$ y $t^{\mu}=\delta_{0}^{\mu}$. Utilizando estas relación conjuntamente con $h_{\mu}^{0}=h^{\mu 0}=0$ podemos escribir las componentes de $g^{\mu \nu}$ como

$$
\left(g^{\mu \nu}\right)=\left(\begin{array}{cc}
-\frac{1}{N^{2}} & -\frac{N^{i}}{N^{2}} \\
-\frac{N^{i}}{N^{2}} & h^{i j}-\frac{N^{i} N^{j}}{N^{2}}
\end{array}\right)
$$

invirtiendo $\left(g^{\mu \nu}\right)$ obtenemos

$$
\left(g_{\mu \nu}\right)=\left(\begin{array}{cc}
-N^{2}+N^{i} N_{i} & -N^{i} \\
-N^{i} & h_{i j}
\end{array}\right)
$$


Haciendo uso de las ecuaciones (18), (19) y (20) podemos encontrar las expresiones para $h_{\mu \nu}, h^{\mu \nu}$ y $h_{\nu}^{\mu}$, las cuales son

$$
\begin{aligned}
\left(h_{\mu \nu}\right) & =\left(\begin{array}{cc}
N^{i} N_{i} & -N^{i} \\
-N^{i} & h_{i j}
\end{array}\right), \\
\left(h^{\mu \nu}\right) & =\left(\begin{array}{cc}
0 & 0 \\
0 & h^{i j}
\end{array}\right), \\
\left(h_{\nu}^{\mu}\right) & =\left(\begin{array}{cc}
0 & 0 \\
-N_{i} & \delta_{j}^{i}
\end{array}\right) .
\end{aligned}
$$

En términos de las variables ADM y con la ayuda de $g_{\mu \nu}$ , la 3-métrica del espacio-tiempo toma la forma siguiente

$$
d s^{2}=\left(-N^{2}+N^{i} N_{i}\right) d t^{2}-2 N_{i} d x^{i} d t+h_{i j} d x^{i} d x^{j} .
$$

Podemos notar que $h_{i j}$ proporciona las distancias entre puntos de $\Sigma_{t}$ con coordenadas $x^{i}$ y $x^{i}+d x^{i}$. Por tanto, la 3-métrica caracteriza la geometría intrínseca de la hipersuperficie $\Sigma_{t}$.

\section{Geometría intrínseca y extrínseca}

La métrica espacial $h_{a b}$ es una cantidad intrínseca y permite definir un único operador derivada covariante $D_{a}$ en $\Sigma_{t}$ tal que $D_{a} h_{b c}=0$. Esta derivada covariante puede ser escrita en términos de la derivada covariante espaciotemporal $\nabla_{a}$ como

$$
D_{c} T_{b_{1} \ldots b_{l}}^{a_{1} \ldots a_{k}}=\left(h_{d_{1}}^{a_{1}} \ldots h_{d_{k}}^{a_{k}} h_{b_{1}}^{e_{1}} \ldots h_{b_{l}}^{e_{l}}\right) h_{c}^{f} \nabla_{f} T_{d_{1} \ldots d_{m}}^{c_{1} \ldots c_{n}} .
$$

Definición. Dada la derivada covariante tridimensional $D_{a}$ podemos definir el tensor de curvatura intrínseca como:

$$
{ }^{(3)} R_{a b c}^{d} w_{d}=D_{a} D_{b} w_{c}-D_{b} D_{a} w_{c}
$$

para toda 1-form espacial, esto es, $w_{a} n^{a}=0$ de esta curvatura intrínseca Riemanniana, podemos obtener el tensor de Ricci ${ }^{(3)} R_{a b}$ y el escalar de curvatura ${ }^{(3)} R$ usando las respectivas contracciones.

\section{Tensor de curvatura extrínseca}

La curvatura extrínseca $K_{\mu \nu}$ se entiende como la proyección de los gradientes de los vectores normales $n_{\mu}$ sobre un folio o rebanada $\Sigma_{t}$, es decir, la curvatura extrínseca nos indica como el vector normal $n_{\mu}$ varía cuando es desplazado de un punto a otro sobre el folio $n_{\mu}$. Dicho de otra manera, el tensor de curvatura extrínseca $K_{\mu \nu}$ es la medida del cambio del vector normal bajo transporte paralelo y nos indica como las hipersuperficies espaciales tridimensionales o folios $\Sigma_{t}$ son inmersas en el espacio-tiempo tetradimensional $M$. Esquematicamente la figura 3, nos muestra la variación de los vectores normales sobre una rebanada espacial.

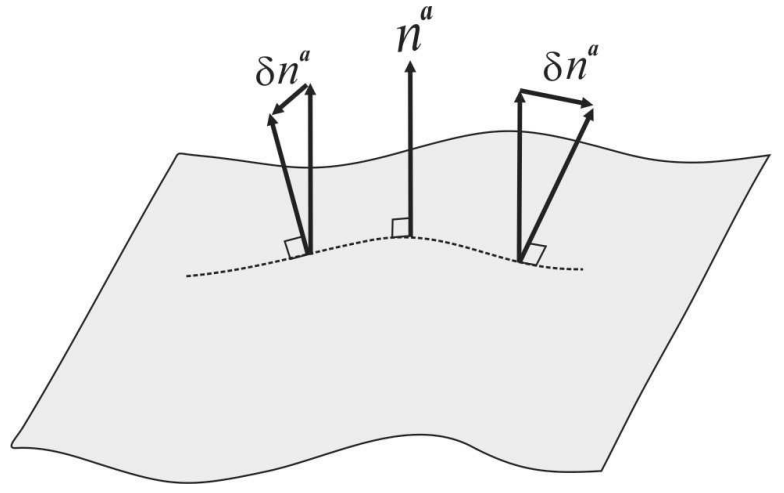

Figura 3: La curvatura extrínseca mide la variación de la dirección de los vectores normales sobre la hipersuperficie tridimensional

Definición. Dado un vector $n^{a}$ normal a la superficie $\Sigma_{t}$, el tensor de curvatura extrínseca es un tensor espacial en $\Sigma_{t}$ dado por

$$
K_{a b}=D_{a} n_{b}=h_{a}^{c} h_{b}^{d} \nabla_{c} n_{d} .
$$

Haciendo uso de la relación (20) encontramos que el tensor de curvatura extrínseca toma la forma

$$
K_{a b}=h_{a}^{c} \nabla_{c} n_{b}+n_{b} n^{d} h_{a}^{c} \nabla_{c} n_{d} .
$$

Haciendo uso de la ecuación anterior se puede demostrar la propiedad simétrica del tensor de curvatura extrínseca $K_{a b}=K_{b a}$. Usando esta relación de simetría, se puede escribir el tensor de curvatura como $K_{a b}=\frac{1}{2}\left(K_{a b}+K_{b a}\right)$ reemplazando la ecuación (20) y efectuando las operaciones apropiadas se obtiene

$$
2 K_{a b}=n^{c} \nabla_{c} h_{a b}+h_{c b} \nabla_{a} n^{c}+h_{a c} \nabla_{b} n^{c} .
$$

Podemos notar que el término de la derecha corresponde a la derivada de Lie de $h_{a b}$ respecto de $n$, por tanto se tiene que

$$
K_{a b}=\frac{1}{2} \mathcal{L}_{n} h_{a b}
$$

Considerando la relación $\mathcal{L}_{N} h_{C D}=D_{a} N_{b}+D_{b} N_{a}$ y usando la ecuación

$$
\dot{T}_{b_{1} \ldots b_{n}}^{a_{1} \ldots a_{n}}=\left(h_{c_{1}}^{a_{1}} \ldots h_{c_{n}}^{a_{n}} h_{d_{1}}^{b_{1}} \ldots h_{d_{m}}^{b_{m}}\right) \mathcal{L}_{t} T_{d_{1} \ldots d_{n}}^{c_{1} \ldots c_{n}},
$$

se obtiene finalmente una expresión final para el tensor de curvatura extrínseco

$$
K_{a b}=\frac{1}{2 N}\left(\dot{h}_{a b}-D_{a} N_{b}+D_{b} N_{a}\right)
$$

donde $\dot{h}_{a b}=\frac{\partial h_{a b}}{\partial t}$. 


\section{Ecuaciones de Gauss, Codazzi y Ricci}

La curvatura intrínseca, medida en la Relatividad General por el tensor de la curvatura de Riemann $R_{\text {efg }}^{h}$ también es descrita en función de $h_{i j}, N$ y $N_{i}$. Para esto debemos tomar tres proyecciones del tensor de Riemann, una proyección espacial completa sobre una hipersuperficie espacial $\Sigma_{t}$, y las otras proyecciones de uno y dos índices en la dirección normal $n^{\mu}$. Estas proyecciones darán origen a las Ilamadas ecuaciones de Gaus, Codazzi y Ricci [8].

Calcularemos las componentes de $R_{e f g}^{h}$ en términos de $K_{a b}$ y ${ }^{(3)} R_{a b c}^{d}$. Para realizar esto aplicamos el operador $D$ a un vector $w_{c}$

$$
D_{b} w_{c}=h_{c}^{d} h_{b}^{e} \nabla_{d} w_{e} .
$$

De esta manera calculamos,

$$
\begin{aligned}
\left(D_{a} D_{b}-D_{b} D_{a}\right) w_{c}= & h_{a}^{f} h_{b}^{d} h_{c}^{e}\left(\nabla_{f} \nabla_{d}-\nabla_{d} \nabla_{f}\right) w_{c} \\
& +\left(K_{b c} K_{a}^{e}-K_{a c} K_{b}^{e}\right) w_{c},
\end{aligned}
$$

por definición, notamos que, el primer término de la izquierda corresponde a ${ }^{(3)} R_{a b c}^{d}$ y el primer término del lado derecho corresponde a ${ }^{(4)} R_{a b c}^{d}$, obteniendo la siguiente expresión

$$
{ }^{(4)} R_{a b c}^{d}={ }^{(3)} R_{a b c}^{d}-K_{b c} K_{a}^{d}+K_{a c} K_{b}^{d}
$$

la cual es conocida como la ecuación de Gauss-Codazzi [1] De igual manera, calculando la relación

$$
h_{e}^{a} h_{f}^{b} h_{g}^{c} R_{a b c d} n^{d}=h_{e}^{a} h_{f}^{b} h_{g}^{c} R_{a b c d} n^{d}\left(\nabla_{a} \nabla_{b}-\nabla_{b} \nabla_{a}\right) n^{c},
$$

se demuestra que

$$
R_{e f g d} n^{d}=D_{e} K_{f g}-D_{f} K_{e g},
$$

podemos notar que la expresión anterior expresa la proyección del tensor $R_{e f g}$ sobre $n^{d}$, escribiendo adecuadamente obtenemos

$$
{ }^{(4)} R_{e f g}^{\perp}=D_{e} K_{f g}-D_{f} K_{e g},
$$

a esta expresión se le conoce como la ecuación de CodazziMainardi [9].

Las ecuaciones de Gauss y Codazzi dependen de la métrica $h_{\mu \nu}$, de la curvatura extrínseca $K_{\mu \nu}$ y de sus derivadas espaciales; estas ecuaciones pueden pueden interpretarse como condiciones de integrabilidad, permitiendo que los folios o rebanadas $\Sigma_{t}$ llenen la variedad $M$.

Para obtener la ecuación de Ricci partimos de su definición

$$
R_{a b}=R_{a c b}^{c}
$$

usando la relación $R_{a b} n^{a} n^{b}=-\left(\nabla_{a} \nabla_{c}-\nabla_{c} \nabla_{a}\right) n^{c} n^{a}$ obtenemos

$$
R_{a b} n^{a} n^{b}=K^{2}-K_{a b} K^{a b}+\triangle_{; a}^{a}
$$

donde $\triangle_{; a}^{a}=\nabla_{a}\left(-n^{a} \nabla_{c} n^{c}+n^{c} \nabla_{c} n^{a}\right)$.

Usando las ecuaciones de Gauss-Codazzi y la ecuación de Ricci obtenemos el escalar de Ricci, que viene expresado por

$$
{ }^{(4)} R={ }^{(3)} R+K_{a b} K^{a b}-K^{2}-2 \triangle_{; a}^{a} .
$$

\section{Formulación hamiltoniana de la Relatividad Gene- ral}

La densidad lagrangiana de Einstein-Hilbert esta expresada por

$$
\mathcal{L}=\sqrt{-g}^{(4)} R=\sqrt{-g}^{(3)} R-K^{2}+K_{a b} K^{a b}+\triangle_{; a}^{a}
$$

usando la relaciones $S=\int d x^{4} \sqrt{-g} R$ y $\sqrt{-g}=N \sqrt{h}$ obtenemos

$$
S=\int N \sqrt{h}\left({ }^{(3)} R+K_{a b} K^{a b}-K^{2}\right) d x^{4} .
$$

Usando la métrica de Witt

$$
G^{a b c d}=\frac{1}{2} \sqrt{h}\left(h^{a c} h^{b d}+h^{a d} h^{b c}-2 h^{a b} h^{c d}\right)
$$

se puede escribir la densidad lagrangiana como

$$
\mathcal{L}=N G^{a b c d} K_{a b} K_{c d}+\sqrt{h}^{(3)} R
$$

Los momentos canónicos vienen dados por

$$
\begin{aligned}
\pi^{a b} & =\frac{\partial \mathcal{L}}{\partial \dot{h}_{a b}}=\sqrt{h}\left(K^{a b}-K h^{a b}\right) \\
\pi_{N} & =\frac{\partial \mathcal{L}}{\partial \dot{h}}=0, \quad \pi_{N^{a}}^{a}=\frac{\partial \mathcal{L}}{\partial \dot{N}^{a}} .
\end{aligned}
$$

Por lo tanto, la densidad hamiltoniana viene dada por la siguiente expresión

$$
\begin{aligned}
\mathcal{H}=\pi^{a b} \dot{h}_{a b}-\mathcal{L}=N G_{a b c d} \pi^{a b} \pi^{c d} & -N \sqrt{N}^{(3)} R \\
& -2 N_{b} D_{a} \pi^{a b}
\end{aligned}
$$

Usando la relación $H=\int d x^{3} \mathcal{H}$ calculamos la hamiltoniana de la Relatividad General, cuya expresion viene dada por

$$
\begin{array}{r}
H=\int d x^{3}\left(\pi^{a b} \dot{h}_{a b}-\mathcal{L}\right)=N G_{a b c d} \pi^{a b} \pi^{c d}-N \sqrt{N}^{(3)} R \\
-2 N_{b} D_{a} \pi^{a b}
\end{array}
$$




\section{Conclusiones}

Se ha descrito el formalismo necesario para comprender la formulación hamiltoniana de la Relatividad General. Hemos derivado todas las ecuaciones necesarias para que el espacio tiempo tetradimensional pueda ser foliado en un conjunto de hipersuperficies espaciales tridimensionales rotuladas con una variable $t$ asociada a una variable temporal. El formalismo hamiltoniano es importante para obtener soluciones numéricas a las ecuaciones de la Relatividad General de Einstein para sistemas físicos reales o complejos, así como es la base para la cuantización canónica de la gra- vedad. Este formalismo, en su version moderna, en analogía con teorías de gauge de Yang-Mills, utiliza las conecciones y holonomías como variables centrales. Las nuevas variables, llamadas variables de Ashtekar, son la base para la gravedad cuántica de lazos [10].

Finalmente, señalamos que la formulacion hamiltoniana es importante para calcular cantidades conservadas quasilocales como la energía, el momento, etc., para sistemas gravitatorios con condición de frontera asintóticamente plana y para modelos cosmológicos homogéneos y anisotrópi$\cos [11$.

\section{Referencias}

[1] M. Alcubierre, Introduction to Numerical Relativity, Oxford University Press, New York (2008).

[2] P. M. A. Dirac,Can. J. Math. 2, 129 (1950).

[3] R. Arnowitt, S. Deser, C. W. Misner,C. W., Gravitation: an introduction to current research, editado por L. Witten, Ed. Wiley and Sons, New York 1962).

[4] J. A. Wheeler, Ann. Phys. 2, 604 (1957).

[5] H. Goldstein, Classical Mechanics, pp.696-703, Third edition, Addison-Wesley, New York (2002).

[6] C. W. Misner, K. S. Thorne, J. A. Wheeler, Gravitation, W. H. Freeman and Copmpany, San Francisco (1973).

[7] R. M. Wald, General Relativity, The University of Chicago Press, Chicago (1984).

[8] M. Smarr, J. W. York, Phys. Rev. D 17, 2529 (1978).

[9] T. W. Baugmarte y S. L. Shapiro, Numerical Relativity. Solving Einstein's Equations on the Computer, Cambridge University Press, New York (2010).

[10] T. Thiemann, Modern Canonical Quantum General Relativity, Cambridge Monographs on Mathematical Physics, Cambridge University Press, New York (2007).

[11] C. C. Chang, J. M. Nester y C. M. Chen, Phys. Rev. Lett. 83, 1897 (1999); J. M. Nester, L. L. So y T. Vargas, Phys. Rev. D 78, 044035 (2008). 